\title{
La eficiencia técnica de los bancos durante la crisis Caso aplicado a la rentabilidad de la banca comercial en México y Chile
}

Werner Kristjanpoller

Rodríguez

Departamento de Industrias, Economía y Negocios, Universidad Técnica Federico Santa María. werner.kristjanpoller@usm.cl

Oscar Saavedra Rodríguez Departamento de Industrias, Economía y Negocios, Universidad Técnica Federico Santa María oscar.saavedra@usm.cl

\section{Resumen}

Este artículo analiza la eficiencia en la generación de rentabilidad de los bancos comerciales que operan en México y Chile, así como su comportamiento en periodo de crisis. En particular, se pretende determinar si en dicho periodo los bancos son más eficientes en la utilización de recursos para generar utilidades sobre los activos totales y sobre su patrimonio que en los periodos normales. La metodología aplicada es el análisis envolvente de datos (DEA, por sus siglas en inglés), introduciendo una innovación en su aplicación ya que se hace una comparación con unidades de diferentes periodos. Finalmente, se puede concluir que existe evidencia que los bancos de mayor eficiencia del periodo completo de análisis se concentran en el desempeño de los periodos de crisis, desde el punto de vista del resultado medido como el retorno sobre activos (ROA, por sus siglas en inglés) y el retorno sobre patrimonio (ROE, por sus siglas en inglés). En la mayoría de los casos, los bancos que alcanzaron mayor eficiencia de utilidad en el periodo previo a la crisis tienen un desempeño menos eficiente al compararlos con los mejores del tiempo de crisis.

Palabras clave: análisis envolvente de datos, eficiencia técnica, bancos, rentabilidad y periodo de crisis.

Clasificación JEL: G21, M21, C67. 


\title{
Banking technical efficiency during crisis periods. Evidence of banks' profita- bility in Mexico and Chile
}

\begin{abstract}
This paper aims to determine evidence about the efficiency of commercial banks under crisis periods when compared against their regular operating periods. Particularly, the Chilean and Mexican banking industries are reviewed by an innovated Data Envelopment Analysis (DEA) methodology. Conclusive evidence of an increase in efficiency for the incumbent banks under crisis periods is presented, as measured by their Return on Assets (ROA) and Return on Equity (ROE). Finally, evidence is found showing that when considering the banks that are more efficient during the whole period of analysis, their better performance is focused during the crisis periods, measured by their ROA and ROE as performance indexes. In most cases, banks that were more efficient during the previous-to-crisis period, had a lower performance during the crisis periods.
\end{abstract}

Keywords: data envelopment analysis, technical efficiency, banks, profitability and crisis period.

JEL Classification: G21, M21, C67

\section{Introducción}

En la actualidad, la economía mundial ha estado expuesta a una fuerte crisis que comenzó en el 2007 con la crisis subprime, aun cuando su inicio formal se dio con el hito de la quiebra de Lehman Brothers en septiembre de 2008. Posteriormente, se ha evidenciado una crisis financiera en alguno de los países miembros de la Comunidad Europea. Toda esta turbulencia financiera y el colapso de algunas instituciones financieras y bancarias ha hecho que exista una mayor atención sobre el sector bancario en el nivel mundial, en especial sobre sus resultados y desempeño, como por ejemplo la eficiencia en el uso de los recursos.

Por otro lado, el uso de los ratios financieros ha sido utilizado por décadas para la evaluación de desempeños de las empresas. Dos son las principales razones que explican el porqué del uso de los ratios como herramientas de análisis; la primera es que permite la comparación entre diferentes compañías de diferentes tamaños; la segunda es que son de gran utilidad para el control de las características del sector. 
Existen ratios financieros para medir la eficiencia de la utilización de recursos. En particular el análisis de ellos se puede medir de dos formas: la primera es observar el aumento de resultados (outputs) a un nivel de recursos (inputs) constante; la segunda es observar la disminución del nivel de recursos para lograr el mismo nivel de resultado. El problema se genera al querer analizar la eficiencia cuando existen variados resultados y diferentes recursos por analizar.

Los métodos de frontera eficiente, tanto paramétricos como no paramétricos, superan al análisis de ratios porque toman en cuenta la interacción entre resultados y recursos de manera simultánea. La idea de este análisis es ver el desempeño relativo de las distintas unidades de producción (DMU), determinando el valor de la eficiencia de resultados con respecto a los recursos utilizados. En particular dentro de estos métodos se encuentra el análisis envolvente de datos (DEA) que permite la determinación, para cada periodo o selección de empresas, de la empresa que tuvo el desempeño más eficiente entre la generación de resultados variados y la utilización de diferentes recursos en una combinación lineal.

Para el análisis de bancos se ha utilizado la eficiencia de costos (cost efficiency) y la eficiencia de generación de utilidad (profit efficiency o PROFEFF). Sin embargo, el objetivo de maximización de la utilidad no implica necesariamente la entrega del servicio al menor costo; por ende, analizar la eficiencia de la obtención de la utilidad es una mejor fuente de información sobre la gestión de los administradores (Maudos et al., 2002). En las organizaciones con fines de lucro, el potencial de generación de rentabilidad relacionado con la eficiencia de la utilidad es importante, así como fundamental entender su descomposición es fundamental Portela y Thanassoulis (2007).

Berger y Mester (1997) demuestran que la eficiencia de costo y la eficiencia de las ventas pueden tener correlación negativa, lo que apunta a que mayores costos pueden ser incurridos para alcanzar mejores resultados, dándole una mayor importancia a la eficiencia de la utilidad por sobre la eficiencia de los costos. De hecho Rogers (1998) encuentra una correlación positiva entre la eficiencia de las ventas y la eficiencia de las utilidades.

En este artículo se analizan el desempeño financiero de los bancos comerciales más importantes en México y en Chile, por medio de un análisis envolvente de datos (DEA), con lo que se distingue la eficiencia en tiempos de crisis y en tiempos de no crisis. Al incorporar un análisis de dos periodos se trata de determinar si los 
patrones de los desempeños de los bancos, con respecto a los recursos utilizados para generar rentabilidad en periodo de crisis, son más eficientes que los patrones óptimos del periodo normal. Las medidas de rentabilidad de los bancos serán la rentabilidad sobre activos (ROA) y la rentabilidad patrimonial (ROE) por ser las más utilizadas en el desempeño bancario (Van Horen, 2007).

La innovación de nuestro trabajo radica en la aplicación del método DEA, ya que se encontrarán los desempeños bancarios más eficientes en la generación de rentabilidad en el periodo previo a la crisis, los cuales se incluirán en el análisis de eficiencia en el periodo de crisis en un análisis para los dos países analizados. El pensamiento previo que se podría tener se refiere a que en los periodos de crisis la administración de los bancos es más ajustada y se plasma en mejores desempeños en la relación de los resultados obtenidos con respecto a los recursos utilizados; la eficiencia de éstos para la generación de resultados en tiempo de crisis para los bancos comerciales analizados es importante porque de esta forma se aumentan las posibilidades de poder sobreponerse a los tiempos adversos.

Este artículo está compuesto por cuatro partes: en la primera se analizan los diferentes estudios que hacen referencia sobre el análisis de eficiencia; luego se describe la metodología utilizada y los mercados estudiados; posteriormente, se aplica la metodología a los mercados analizados con lo que se obtiene los resultados sobre la eficiencia; por último, se presentan las principales evidencias e implicancias del estudio.

\section{Revisión de la literatura}

Un análisis más exacto de eficiencia de empresas componentes de una industria a partir de los ratios financieros se puede realizar a través de los métodos de frontera, ya sea paramétricos o no paramétricos, dados que éstos presentan la ventaja de medir la interacción simultánea entre entradas y resultados y de medir relativamente la eficiencia entre las distintas unidades analizadas. La metodología de análisis envolvente de datos (DEA) la comienza a desarrollar Farrell (1957) y sus aplicaciones han sido múltiples.

El principal objetivo del enfoque de frontera es la determinación de un valor de eficiencia relativa asociado a un periodo de análisis y un ranking para las unidades de producción, pudiéndose identificar aquellas áreas que no están siendo eficientes en resultado y/o entrada en cada unidad. 
En la literatura hay un número similar de estudios de eficiencia para métodos paramétricos como para no paramétricos; sin embargo realmente no hay consenso sobre un método preferido para determinar las mejores prácticas en el uso de una frontera cuya eficiencia relativa es medida (Berger y Humphrey 1997). Está aceptado que los enfoques paramétricos imponen una forma de funcionamiento particular que presupone la forma de la frontera. Por lo general, especifican un análisis local, pero proporciona pobres aproximaciones para la data bancaria, que no están cerca de la misma escala y cartera de productos (McAllister y McManus, 1993).

Los estudios no paramétricos imponen menos estructura en la frontera; entre ellos el DEA ha llegado a ser uno de los mejores métodos de evaluación de eficiencia en términos tanto de número de publicaciones como aplicaciones en el mundo real (Golany, 1988).

Sathye (2001) indagó la eficiencia en los bancos australianos versus la eficiencia mundial en la década de los años noventa con la base de la metodología DEA, llegando a la conclusión de que los bancos australianos tienen una menor eficiencia en relación con la eficiencia promedio del resto del mundo y con ello un déficit en sus índices de buenas prácticas.

Por su parte, Jemric y Vujcic (2002) investigaron la eficiencia de los bancos croatas a través de la metodología DEA llegando a la conclusión de que los bancos extranjeros eran más eficientes que los locales y que los bancos locales nuevos eran más eficientes que los antiguos. De igual forma, Sathye (2003) investigó la eficiencia de los bancos de India, separándolos en dos tipos de análisis focalizados hacia los recursos; el primero orientado hacia el análisis de los gastos y el segundo sobre el análisis de los activos, concluyendo que el sector bancario público indio tiene una mayor eficiencia promedio que el sector bancario privado. El sector público de bancos presenta una menor eficiencia que los extranjeros si se orientan las entradas hacia los activos, pero sigue siendo mayor que los privados.

Diversos estudios se han realizado para determinar eficiencia de los DMU utilizando variables enfocadas a los costos y beneficios (Ariff y Can, 2008; Maudos et al., 2002; Clark y Siems, 2002; Färe, Grosskopf y Weber, 2004; Rogers, 1998). Se tienen estudios como el de Maudos et al. (2002) que se enfoca en las diferencias y relaciones con respecto a la eficiencia entre los costos y beneficios utilizando el retorno sobre los activos (ROA) como variable. También existen otros estudios que se enfocan en medidas de beneficio para probar la consolidación de bancos 
pequeños (Akhigbe y McNulty et al., 2001; Hannan, 1991; Purroy y Salas, 2000; Rhoades, 1980), los cuales sugieren mediciones centradas en los beneficios de las instituciones bancarias para medir eficiencia (PROFEFF) centrando distintas teorías a través de la historia para, así, establecer que la eficiencia dentro de este tipo de instituciones es vital para la consolidación y supervivencia de estas últimas.

Desde el punto de vista de la aplicación existe una serie de estudios basados en la metodología DEA para el análisis de la industria bancaria tanto en bancos (Haslem et al. 1999; Mukherjee et al. 2001; Saha y Ravishankar, 2000; Halkos y Salamoulis, 2004), como también a nivel filiales (Athanassopoulos y Giokas, 2000; Drake y Howcroft, 1994; Sevcovic et al., 2001; Sherman y Ladino, 1995), como en los trabajos de Vassiloglou y Giokas (1990).

Aunque no existen modelos aceptados para la interpretación de la tecnología en donde se proveen los servicios y productos del banco como entrada/resultado, Dekker y Post (2001) enuncian que la tecnología puede ser representada por ciertos resultados en forma de préstamos, hipotecas y seguros, los cuales son producidos por ciertos entradas como el personal, computadores, etcétera.

Dada la multitud de entradas y resultados que pueden usarse con base en esta metodología, Ozkan-Gunay y Tektas (2006) afirman que la elección de éstos es vital porque se pueden llegar a diferentes conclusiones en puntuación y ranking.

\section{Metodología y datos}

En este estudio se utiliza un método no-paramétrico para evaluar el desempeño de las corporaciones bancarias. Específicamente se usa la metodología DEA, el cual es un método no estadístico que usa programación lineal, entregando una medida de eficiencia técnica relativa de diferentes unidades de producción (DMU) que hagan una tarea igual o similar. El enfoque de análisis envolvente de datos fue introducido por Farrell (1957) para obtener como resultado la eficiencia productiva, así como también sus componentes, la eficiencia asignativa y la eficiencia técnica. Este enfoque fue desarrollado y ampliado por Charnes et al. (1978) y posteriormente por Färe et al. (1982). Un completo de análisis sobre la evolución, desarrollo y mejoras del modelo se encuentra en Seiford (1996).

La principal ventaja de esta técnica es que se puede tratar el caso de múltiples entradas y resultados, así como los factores que no pueden ser controlados por una 
administración. La aplicación de esta técnica facilita la comparación de eficiencia para una muestra grande de bancos con el uso simultáneo del mismo criterio para determinar la eficiencia de cada banco.

La metodología DEA compara los niveles de entrada y el resultado de todos los DMU en un análisis en conjunto y define una frontera eficiente identificando la mejor práctica relativa de estos DMU. La mejor práctica se define como el proveedor de servicios, que utiliza la menor cantidad de recursos para proporcionar el volumen y cartera de servicios iguales o superiores a la norma de calidad de los negocios (Sherman y Ladino 1995). Las unidades de mejores prácticas, relativamente eficientes, son identificadas por la clasificación DEA de productividad de un $100 \%(\mathrm{E}=1)$. Las unidades ineficientes son identificadas por una clasificación $\mathrm{E}<1$.

Una unidad con ineficiencia relativa implica la existencia de unidades que tienen una mayor eficiencia en el conjunto de datos de unidades analizadas, pudiéndose determinar el monto de recursos en exceso utilizado por cada unidad ineficiente, así como la cantidad de exceso de capacidad para aumentar los servicios resultados en estas unidades sin utilizar recursos adicionales.

Para ilustrar la metodología, se ejemplificará con el análisis de eficiencia de ocho bancos comerciales $\left(T_{1}, T_{2}, \ldots, T_{8}\right)$, mediante dos ratios distintos, los gastos de apoyo sobre total de activos (GATA) como entrada y la rentabilidad patrimonial (ROE) como resultado. Se puede observar en la figura 1 que los bancos que obtuvieron una eficiencia óptima son el $T_{1}, T_{2}, T_{3}$ y $T_{4}$. La frontera eficiente se determina por los segmentos que unen los puntos entre estos cuatri bancos. El banco $T_{5}$ no está en la frontera y se considera como no eficiente. El punto $T_{u}$ determina el nivel óptimo de eficiencia y representa la combinación entre los ratios $R_{l}$ y $R_{2}$ en la misma proporción que el banco $T_{5} \mathrm{y}$, por lo tanto, se considera un punto de referencia para éste, calculándose la eficiencia del banco $T_{5}$ con respecto a $T_{u}$. Cabe destacar que $T_{u}$ es una combinación lineal de $T_{2}$ y $T_{3}$, siendo el conjunto de referencia para el banco $T_{5} \operatorname{los}$ bancos $T_{2}$ y $T_{3}$. La medida en que $T_{u}$ excede a $T_{5}$ es el tamaño de la ineficiencia. El grado de eficiencia para el banco $T_{5}$ se encuentra en el ratio $\mathrm{O} T_{5} / \mathrm{O}$ $T_{u}$ como se muestra en la figura 1 . 
Figura 1

Presentación gráfica del modelo DEA

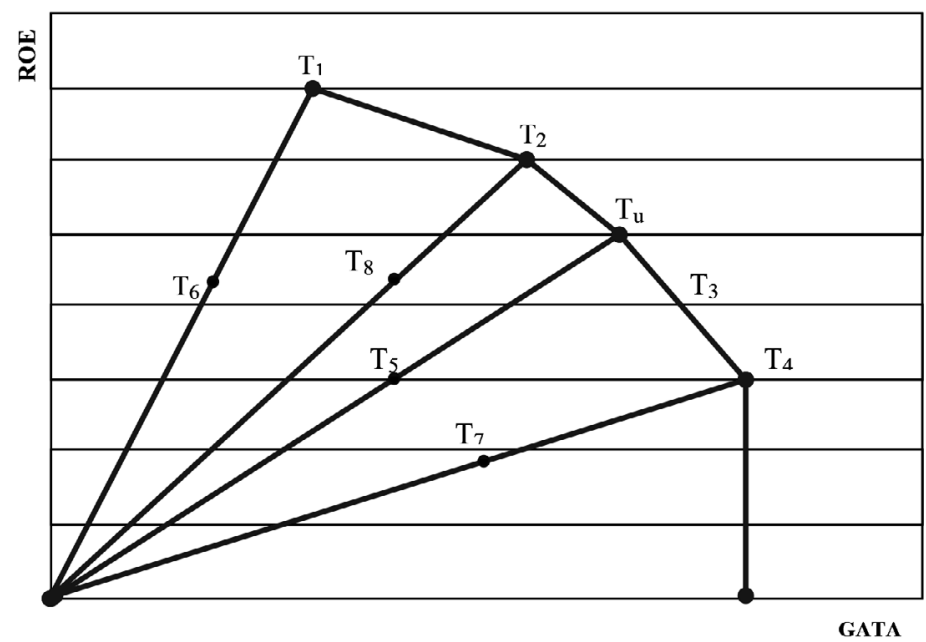

Charnes et al. (1978) plantearon un modelo matemático para la determinación de la eficiencia a través del modelo DEA, formulándolo como una programación lineal donde la función objetivo es la maximización de los resultados sobre las entradas, sujeto a que esta función objetivo no pueda superar el valor 1 al hacer el análisis individualmente, el cual se puede observar en el modelo1.

$$
\operatorname{Max} E_{0}=\frac{\sum_{i=1}^{k} u_{i} y_{i 0}}{\sum_{j=1}^{m} v_{j} x_{j 0}}
$$

Sujeto a

$$
\begin{gathered}
\frac{\sum_{i=1}^{k} u_{i} y_{i r}}{\sum_{j=1}^{m} v_{j} x_{j r}} \leq 1 r=1, \ldots, N \\
u_{i}, v_{j} \geq \varepsilon, i=1, \ldots, k j=1, \ldots, m 0<\varepsilon \ll 1
\end{gathered}
$$

donde $y_{i r}$ y $x_{j r}$ son los niveles de resultados y entradas del banco $\mathrm{r}$ respectivo, $u_{i}$ y $v_{j}$ son los pesos de los resultados y entradas dentro del modelo, $\varepsilon$ es un número positivo muy pequeño utilizado como un límite inferior para los pesos de las entradas y resultados. $E_{0}$ es el ratio de los pesos de los resultados sobre los pesos de las entradas. 
Debido a la dificultad para resolver el modelo 1 se utiliza su modelo equivalente lineal (modelo 2), también generado por Charnes et al. (1978). Este modelo 2 es la linealización del 1; la transformación se basa en la restricción inicial del modelo 1 , la cual plantea que la función objetivo debe ser menor o igual a 1; en este caso, la función objetivo se centra en la valoración de los resultados de las DMU; debe ser menor o igual a la valoración de las entradas o recursos utilizados de estas unidades, así se linealiza la función objetivo y las restricciones del problema.

$$
\begin{gathered}
\operatorname{Max} E_{0}=\sum_{i=1}^{k} u_{i} y_{i 0} \\
\sum_{i=1}^{k} u_{i} y_{i r} \leq \sum_{j=1}^{m} v_{j} x_{j r} \quad r=1, \ldots, N \\
u_{i}, v_{j} \geq \varepsilon, i=1, \ldots, k j=1, \ldots, m 0<\varepsilon \ll 1
\end{gathered}
$$

La formulación dual de este modelo es útil para hacer un análisis detallado de las unidades ineficientes y tomar acciones correctivas para mejorar su eficiencia; éste queda reflejado en el modelo 3, el cual es la representación en otras dimensiones del modelo 2, invirtiendo los coeficientes del modelo y con ellos transformándolos. El modelo 3 se valida, pues entrega los mismos resultados que su modelo original (modelo 2), pero con una resolución más sencilla.

Sujeto a

$$
\operatorname{Min} Z_{0}-\varepsilon\left(\sum_{j=1}^{m} S_{j}^{-}+\sum_{i=1}^{k} S_{i}^{+}\right)
$$

$$
\begin{gathered}
\sum_{r=1}^{N} \lambda_{r} x_{j r}=Z_{0} x_{j r 0}-S_{j}^{-} \quad j=1, \ldots, m \\
\sum_{r=1}^{N} \lambda_{r} y_{i r}=y_{i r 0}+S_{j}^{+} \quad i=1, \ldots, k \\
\lambda_{r} \geq 0 \quad r=1, \ldots, N \\
S_{j}^{-}, S_{i}^{+} \geq 0 \quad \text { Para todo } j, i \\
Z_{0} \text { Irrestricto }
\end{gathered}
$$


donde $Z_{0}$ es el factor de eficiencia radial que muestra la posible reducción de entradas para el banco $0, \lambda_{r}$ muestra la contribución del banco $r$ en la obtención de la eficiencia del banco 0 clasificado; $S_{j}^{-}$y $S_{j}^{+}$son las variables de holgura que muestran los entradas extra que se podrían guardar y los resultado extra que se podrían ganar en r. Los valores positivos de las variables de holgura en la solución óptima significa que los correspondientes entrada y resultado del banco 0 pueden mejorar aún más, después de que los niveles de entrada han sido contrastados por la proporción $Z_{0} *$ (Thanassoulis, 2003). Si $Z_{0}^{*}=1$ y las variables de holgura son 0 , entonces el banco en cuestión es eficiente porque el modelo no puede encontrar otro punto óptimo en donde se pueda mejorar la gestión de los entrada y resultado (Thanassoulis, 2003).

Los datos utilizados para este estudio corresponden a bancos comerciales con resultados positivos que operaron en México y en Chile. El periodo de análisis son los años entre 2002 y 2010 y la información fue obtenida desde Superintendencia de Bancos e Instituciones Financieras para el caso de los bancos en Chile (www. sbif.cl), mientras que para el caso de los bancos en México fue la Comisión Nacional Bancaria y de Valores de México (http://www.cnbv.gob.mx). En el Anexo 1 está el listado de los bancos analizados.

La aplicación del DEA en este estudio tiene una innovación con respecto a la forma tradicional de utilizarlo, dado que lo que se busca demostrar es que en tiempo de crisis los bancos son más eficientes; para ello primeramente se analizará la eficiencia de los bancos para el periodo definido como precrisis (2002-2006) para cada país por separado, obteniéndose así para cada año el(los) banco(s) eficiente(s). Luego, para cada país por separado, se forma un grupo con todos los bancos eficiente del periodo precrisis, llamado Grupo de los bancos con buenas prácticas, al cual se le aplicará nuevamente el DEA para encontrar a los bancos más eficientes del periodo precrisis. Posteriormente, se hace el análisis de eficiencia para cada año de crisis (2008-2010), pero en cada año se incluye los bancos más eficientes del periodo precrisis para así poder observar si siguen siendo los más eficientes.

Los ratios por analizar en este estudio están orientados a la generación y cálculo de la rentabilidad de una organización bancaria. Los ratios utilizados de entradas son IPSC, GATA, y los de resultados son ROE y ROA. A continuación se describe la forma de cálculo de cada ratio. 
1. Intereses y cargas asimiladas (IPSC). Es la suma de los gastos por intereses del ejercicio del Estado de Resultado.

IPSC $=$ Gastos por intereses y reajustes + Gastos por comisiones + Pérdida neta de operaciones financieras + Pérdida de cambio neta

2. Gastos de apoyo sobre total de activos (GATA). Este ratio muestra la relación entre los gastos de apoyo (remuneración $(L)$ y administración $(A)$ ) sobre el total de activos.

$$
G A T A=\frac{L_{t}+A_{t}}{T A_{t}}
$$

3. Rentabilidad sobre Patrimonio o Rentabilidad Patrimonial (ROE). Este ratio es el cociente entre las utilidades antes de impuesto sobre el patrimonio promedio. Este ratio estima la eficiencia en que el banco explota su capital.

$$
\frac{R O E_{t}=P B T_{t}}{\left(E_{t}+E_{t^{-1}}\right) / 2}
$$

donde $P B T$ son las utilidades (pérdidas) antes de impuesto, E el patrimonio y $t$ el tiempo.

4.-Rentabilidad sobre activos (ROA). Este ratio es el cociente entre las utilidades antes de impuesto sobre el total de activos. Este índice estima la eficiencia del uso de los activos para la generación de utilidades.

$$
R O A_{t}=\frac{P B T_{t}}{\left(T A_{t}+T A_{t-1}\right) / 2}
$$

donde $P B T$ son las utilidades antes de impuesto, TA el total de activos y t el tiempo.

Dada la orientación del estudio hacia la rentabilidad de las instituciones bancarias, se establece que los IPSC y GATA son determinantes al momento de analizar ROA y ROE dado que un aumento de uno de estos gastos debiese implicar un aumento en la rentabilidad; de no ser así la DMU en cuestión no estaría siendo eficiente. 


\section{Análisis de resultados}

Los resultados obtenidos al aplicar la eficiencia técnica global utilizando el enfoque DEA-CCR serán presentados por país y por índice de resultado; es decir, ROA y ROE. Durante el periodo de análisis la economía mexicana se puede caracterizar por ser una economía orientada a las exportaciones, donde más del $90 \%$ de ellas se encuentra relacionado con algún tipo de tratado de libre comercio con más de 40 países. Gran parte de sus importaciones y exportaciones están relacionadas con sus socios comerciales de Norteamérica. Desde el año 2000 la economía mexicana se ha caracterizado por presentar un crecimiento sostenido moderado medido a través de su PIB, aunque la crisis económica mundial le afectó manifestándose en una desaceleración en el 2008 y una contracción del 6.2\% de su PIB en 2009. Durante 2010 se comienza a consolidar la recuperación, teniendo una tasa de crecimiento del 5.2\%. La inflación se mantuvo controlada, en niveles de $3.3 \%$ al 5.2\%, en torno al valor meta de $4.5 \%$ salvo en 2008 , el cual tuvo una inflación del $6.5 \%$. La tasa de desempleo registra un aumento a partir de 2002 , de $2.74 \%$ hasta $5.35 \%$, en 2010 ; se presenta un incremento acelerado desde agosto de 2008. En cuanto a su moneda, el peso mexicano sufrió una depreciación importante durante septiembre de 2008, lo cual puede ser explicado dentro del contexto de la crisis económica mundial. En anexo 4 se puede observar las gráficas de la evolución del tipo de cambio del peso mexicano con respecto al dólar estadounidense, el crecimiento del PIB mexicano y la inflación anual de México.

Para el caso mexicano, durante el periodo total, se analizaron 21 bancos (anexo 1), que se denotan como DMU1 hasta DMU18, sin guardar relación con el orden presentado en el anexo 1. El primer análisis realizado fue obtener los DMU eficientes para cada año previo a la crisis (2002-2006) y luego obtener los bancos más eficientes llegando a dos DMU para el caso del ROA y cuatro DMU para el caso del ROE, los cuales se presentan en el cuadro 1. 


\section{Cuadro 1 \\ DMU eficientes para el caso mexicano periodo previo a la crisis} (2002-2006)

\begin{tabular}{c|c|c|c}
\hline Resultado & \multicolumn{3}{c}{ ROA } \\
\hline DMU & Eficiencia & DMU & Eficiencia \\
DMU1 (2002) & 0.414 & DMU2 (2002) & $\mathbf{1 . 0 0 0}$ \\
DMU2 (2002) & 0.355 & DMU3 (2002) & 0.967 \\
DMU3 (2002) & 0.219 & DMU4 (2003) & $\mathbf{1 . 0 0 0}$ \\
DMU4 (2003) & 0.199 & DMU5 (2003) & $\mathbf{1 . 0 0 0}$ \\
DMU5 (2003) & 0.269 & DM1 (2004) & 0.230 \\
DMU6 (2004) & $\mathbf{1 . 0 0 0}$ & DMU13 (2004) & 0.647 \\
DMU2 (2005) & $\mathbf{1 . 0 0 0}$ & DMU4 (2004) & 0.640 \\
DMU4 (2006) & 0.076 & DMU17 (2004) & 0.857 \\
DMU7 (2006) & 0.125 & DMU13 (2005) & 0.719 \\
DMU3 (2006) & 0.064 & DMU2 (2005) & $\mathbf{1 . 0 0 0}$ \\
& & DMU17 (2004) & 0.356 \\
& & DMU13 (2006) & 0.692 \\
& & DMU7 (2006) & 0.712 \\
& & DMU3 (2006) & 0.455 \\
\hline
\end{tabular}

Se puede observar que solamente el DMU2 en 2005 fue eficiente en el uso de sus entradas tanto para el ROA y el ROE como salida, siendo el único DMU que aparece eficiente en el análisis de ambos resultados.

Estos bancos eficientes en rentabilidad se incorporaron en el análisis de eficiencia del periodo de crisis (2008-2010) según el caso de análisis de resultados. Se omitió el año 2007 porque es un año de transición de normalidad a crisis. Al hacer el análisis de eficiencia en el periodo de crisis para el caso del ROA, sólo una DMU de las eficientes en precrisis se mantuvo eficiente y la otra perdió su categoría de mayor eficiencia; mientras que para el caso del ROE, dos de las cuatro DMU perdieron su categoría de eficiente en el periodo de crisis. Los resultados se pueden observar en el cuadro 2 . 


\section{Cuadro 2}

DMU eficientes para el caso mexicano periodo de crisis (2008-2010)

\begin{tabular}{|c|c|c|c|c|c|c|c|c|}
\hline \multirow[t]{2}{*}{ Resultado } & \multicolumn{4}{|c|}{ ROA } & \multicolumn{4}{|c|}{ ROE } \\
\hline & DMU & 2008 & 2009 & 2010 & DMU & 2008 & 2009 & 2010 \\
\hline & DMU1 & 0.092 & 0.184 & 0.160 & DMU1 & 0.107 & 0.193 & 0.169 \\
\hline & DMU3 & 0.051 & 0.062 & 0.050 & DMU3 & 0.402 & 0.359 & 0.341 \\
\hline & DMU4 & 0.222 & 0.227 & 0.326 & DMU4 & 0.210 & 0.150 & 0.253 \\
\hline & DMU5 & 0.229 & 0.203 & 0.255 & DMU5 & 0.193 & 0.153 & 0.194 \\
\hline & DMU6 & 0.189 & N/A & N/A & DMU6 & 0.213 & N/A & N/A \\
\hline & DMU7 & 0.073 & 0.046 & 0.034 & DMU7 & 0.059 & 0.235 & 0.202 \\
\hline & DMU8 & 0.318 & 0.454 & 0.503 & DMU8 & 0.042 & 0.062 & 0.066 \\
\hline & DMU9 & 0.685 & 0.539 & 0.673 & DMU9 & 0.118 & 0.103 & 0.112 \\
\hline & DMU10 & 0.537 & 0.678 & 0.594 & DMU10 & 0.076 & 0.108 & 0.136 \\
\hline & DMU11 & 0.133 & N/A & 0.221 & DMU11 & 0.041 & N/A & 0.006 \\
\hline & DMU12 & 0.067 & 0.431 & 0.045 & DMU12 & 0.229 & 0.121 & 0.144 \\
\hline & DMU13 & 0.059 & 0.095 & 0.056 & DMU13 & 0.470 & 0.555 & 0.392 \\
\hline & DMU14 & 0.408 & 0.298 & 0.378 & DMU14 & 0.101 & 0.068 & 0.101 \\
\hline & DMU15 & 0.050 & 0.034 & 0.039 & DMU15 & 0.132 & 0.096 & 0.103 \\
\hline & DMU16 & 1.000 & 1.000 & 1.000 & DMU16 & 1.000 & 1.000 & 1.000 \\
\hline & DMU17 & 0.003 & 0.009 & 0.016 & DMU17 & 0.006 & 0.053 & 0.103 \\
\hline & $\begin{array}{l}\text { DMU6 } \\
\text { (2004) }\end{array}$ & 0.725 & 0.850 & 0.841 & DMU2 (2002) & 1.000 & 1.000 & 1.000 \\
\hline & DMU2(2005) & 1.000 & 1.000 & 1.000 & DMU4 (2003) & 1.000 & 0.819 & 0.937 \\
\hline & & & & & DMU5 (2003) & 0.911 & 1.000 & 1.000 \\
\hline & & & & & DMU2(2005) & 1.000 & 1.000 & 1.000 \\
\hline
\end{tabular}

Interesante es el hecho que la DMU16 es eficiente durante el periodo de crisis para ambos resultados durante todos los años analizados.

Por último, se hace un análisis general con todos los DMU del periodo de crisis de todos los años y los eficientes previo a la crisis como un solo periodo, lo que se encontró son los mismos resultados que si se hubieran realizado año por año; esto significa que hubo DMU eficientes en precrisis, las cuales posteriormente, en 
periodo de crisis, son superadas por otras DMU. También es interesante notar que la eficiencia relativa no cambia al hacerlo por año en crisis o todo como un solo intervalo de tiempo, con lo cual se puede concluir que la función óptima de entradas y resultados es la misma y constante en el tiempo. Para observar los resultados de este último análisis ver anexo 2.

La economía chilena se caracteriza por la apertura de sus mercados, la cual está orientada a la exportación. Chile posee acuerdos comerciales con países representando a más del $90 \%$ de la población mundial, lo que lo cataloga como uno de los países con mayor apertura económica del mundo. Su principal producto de exportación es el cobre, que representa el 35\% de sus exportaciones; además, posee una economía diversificada orientada a servicios, el sector industrial y agroindustrial.

Entre 2002 y 2010 Chile tuvo un crecimiento promedio de su PIB de $3.79 \%$ anual durante el periodo. La crisis económica mundial tuvo repercusiones en Chile, lo que puede observarse en la desaceleración de 2007 y 2008 y la contracción del PIB de 2009, alcanzando una variación de $-1.68 \%$. La inflación en Chile se ha mantenido contenida dentro del valor meta del $3 \%$, la cual fue sobrepasada especialmente durante 2008 y 2009 con un $8 \%$ y un 5.5\% anual respectivamente. La tasa mensual de desempleo se ha mantenido estable con tendencia a la baja teniendo un rango entre el 6\% alcanzado a fines de 2006 y con un máximo de $11.2 \%$ alcanzado a mediados de 2004. La crisis económica mundial tuvo un impacto al aumentar la tasa de desempleo de un 7\% a fines de 2007 hasta llegar a un 10.8\% a mediados de 2009. En cuanto a su moneda, el peso chileno, se aprecia consistentemente desde 2004 hasta inicios de 2008, alcanzando un valor de 435 CLP/USD. Posteriormente, relacionado con la crisis económica mundial sufre una fuerte depreciación a fines del mismo año hasta alcanzar una cotización cercana a los 700 CLP/USD. Desde fines de 2009 esta cotización no supera los 550 CLP/USD, apreciación relacionada con la reactivación económica ocurrida. En el anexo 5 se puede observar las gráficas de la evolución del tipo de cambio del peso chileno con respecto al dólar estadounidense, el crecimiento del PIB chileno y la inflación anual de Chile.

Para el caso chileno, durante el periodo total, se analizaron doce bancos (anexo 1), los cuales se denotan como DMU1 a DMU12 sin guardar relación con el orden presentado en el anexo 1. Al igual que en el caso mexicano, el primer análisis realizado fue obtener los DMU eficientes para cada año previo a la crisis (2002-2006) y los bancos más eficientes precrisis con lo que se obtuvo tres 3 DMU para el caso del ROA y para el caso del ROE, los cuales se presentan en el cuadro 3. 


\section{Cuadro 3 \\ DMU eficientes para el caso chileno periodo previo a la crisis} (2002-2006)

\begin{tabular}{ccc|cc}
\hline Resultado & \multicolumn{2}{c|}{ ROA } & & ROE \\
\hline & DMU & Eficiencia & DMU & Eficiencia \\
\cline { 2 - 5 } & DMU 2 (2002) & 0.848 & DMU 2 (2002) & $\mathbf{1 . 0 0 0}$ \\
& DMU 11 (2002) & 0.642 & DMU 5 (2003) & 0.916 \\
DMU 3 (2003) & 1.000 & DMU 7 (2003) & $\mathbf{1 . 0 0 0}$ \\
DMU 7 (2003) & 1.000 & DMU 2 (2004) & 0.840 \\
DMU 2 (2004) & 0.717 & DMU 5 (2004) & 0.821 \\
DMU 3 (2004) & 0.831 & DMU 2 (2005) & 0.844 \\
DMU 2 (2005) & 0.732 & DMU 3 (2005) & $\mathbf{1 . 0 0 0}$ \\
DMU 3 (2005) & 1.000 & DMU 7 (2005) & 0.848 \\
DMU 11 (2005) & 0.744 & DMU 2 (2006) & 0.957 \\
DMU 2 (2006) & 0.804 & DMU 6 (2006) & 0.914 \\
DMU 6 (2006) & 0.658 & & \\
DMU 11 (2006) & 0.970 & & \\
\hline
\end{tabular}

Se puede observar que DMU7 (2003) y DMU3 (2005) son las únicas eficientes en el uso de sus entradas tanto para el ROA y el ROE como salida, siendo las dos únicas DMU las que aparecen eficientes en el análisis de ambos resultados.

Estos bancos eficientes se incorporaron en el análisis de eficiencia del periodo de crisis (2008-2010), según el caso de análisis de resultados. Al hacer el análisis de eficiencia en el periodo de crisis para el caso del ROA, sólo una DMU de las eficientes en precrisis se mantiene eficiente y las otras pierden su categoría de mayor eficiencia para 2009 y 2010, mientras que para el caso del ROE, las tres DMU perdieron su categoría de eficiente en 2010. Los resultados se pueden observar en el cuadro 4. 
Cuadro 4

DMU eficientes para el caso chileno periodo de crisis (2008-2010)

\begin{tabular}{ccccc|cccc}
\hline Resultado & \multicolumn{3}{c}{ ROA } & & \multicolumn{3}{c}{ ROE } \\
\hline DMU & $\mathbf{2 0 0 8}$ & $\mathbf{2 0 0 9}$ & $\mathbf{2 0 1 0}$ & DMU & $\mathbf{2 0 0 8}$ & $\mathbf{2 0 0 9}$ & $\mathbf{2 0 1 0}$ \\
\hline & DMU 1 & 0.263 & 0.025 & 0.371 & DMU 1 & 0.529 & 0.666 & 0.376 \\
DMU 2 & 0.477 & 0.495 & 0.989 & DMU 2 & 0.742 & 1.000 & 1.000 \\
DMU 3 & 0.351 & 0.468 & 0.950 & DMU 3 & 0.477 & 0.784 & 1.000 \\
DMU 4 & 0.345 & 0.344 & 0.411 & DMU 4 & 0.562 & 0.550 & 0.381 \\
DMU 5 & 0.401 & 0.359 & 0.535 & DMU 5 & 0.720 & 0.637 & 0.469 \\
DMU 6 & 0.526 & 0.658 & 1.000 & DMU 6 & 0.803 & 0.985 & 0.888 \\
DMU 7 & 0.211 & 0.175 & 0.636 & DMU 7 & 0.395 & 0.460 & 0.757 \\
DMU 8 & N/A & 0.082 & 0.172 & DMU 8 & N/A & 0.116 & 0.168 \\
DMU 9 & 0.206 & 0.263 & 0.255 & DMU 9 & 0.526 & 0.569 & 0.309 \\
DMU 10 & 0.355 & 0.334 & 0.566 & DMU 10 & 0.406 & 0.463 & 0.470 \\
DMU 3 (2003) & 1.000 & 0.650 & 0.644 & DMU 2 (2002) & 1.000 & 1.000 & 0.586 \\
DMU 7 (2003) & 1.000 & 0.737 & 0.737 & DMU 7 (2003) & 1.000 & 1.000 & 0.974 \\
DMU 3 (2005) & 1.000 & 1.000 & 1.000 & DMU 3 (2005) & 1.000 & 1.000 & 0.560 \\
\hline
\end{tabular}

Interesante es el hecho que la DMU3 (2005) es la única que mantiene su eficiencia en el caso ROA para el año 2010 y todas las demás pierden esta categoría tanto para el análisis del ROA como del ROE, apoyando la hipótesis de que los bancos son más eficientes en tiempos de crisis. Otro resultado interesante es que tanto la DMU2 y la DMU3 fueron eficientes en el periodo previo a la crisis analizado como ROE, y en el año 2010 pierden esa categoría por sus resultados 2002 y 2005, respectivamente, pero pasan a ser la más eficientes por su desempeño de 2010.

Para finalizar, se hace un análisis general con todos los DMU del periodo de crisis de todos los años y los eficientes previo a la crisis, como un solo periodo, encontrándose sólo los mismos resultados para las DMU que en 2010; es decir, el 2010 fue el año de mayor eficiencia de todos los años analizados. Para observar los resultados de este último análisis ver anexo 3. 


\section{Conclusiones}

Luego del análisis envolvente de datos (DEA) realizado a los bancos comerciales que operaron en el periodo analizado (2002-2010) en México y que operaron en Chile - en la que se realizó una división en dos periodos precrisis (2002-2006) y crisis (2008-2010) - se observa que los desempeños de los bancos analizados son mejores en el último periodo. En particular, esta conclusión se obtiene al haber analizado la generación de rentabilidad, ROA y ROE, con respecto al uso de recursos, siendo mejores los resultados de rentabilidad sobre recursos en los desempeños obtenidos para ambos países por separado en el periodo (2008-2010) que en el periodo previo, entendiendo con esto una mayor eficiencia de utilidad de los bancos en la generación de rentabilidad.

Al hacer el análisis con los datos de todos los desempeños bancarios del periodo de estudio se obtiene que para el caso chileno en el año 2010 se encuentran los desempeños más eficiente desde el punto de vista de generación de rentabilidad, tanto para el ROE como el ROA. Para el caso mexicano, se puede concluir que en el caso de la eficiencia en la generación del ROE, el 2008 fue un año muy eficiente, mientras que en el ROA se encuentra eficiencia mayor al periodo previo a la crisis en cada año de crisis. También en el caso mexicano resalta que la DMU2 (2005) mantuviera su eficiencia en la crisis medida como ROA y ROE, en tanto que la DMU16 (2008) aparece como una unidad eficiente en periodo de crisis también para ambos resultados.

Un resultado interesante es el caso del análisis del ROE para los bancos comerciales en Chile, donde todos los desempeños eficientes en el periodo previo a la crisis fueron superados en su condición de eficiencia por el desempeño obtenido en 2010 por dos de los mismos bancos, ilustrando una mayor eficiencia de la misma unidad de decisión. El caso es de las DMU2 y DMU3, las que fueron eficientes en el análisis de ROE en el periodo analizado previo a la crisis, particularmente en 2002 y 2005, respectivamente, mientras que 2010 pierden esa categoría de eficiente porque tuvieron un desempeño mucho más eficiente.

Para la administración de empresas y los aspectos contables, este artículo demuestra la importancia de la medición de los resultados y control de la gestión en los bancos; además, a través del DEA se puede comparar la eficiencia de una industria y se puede obtener conclusiones para cada una de las DMU componentes y la ra- 
zón de su ineficiencias, lo que significa que pueden mejorar estas DMU ineficientes y cuáles son las mejores prácticas de las empresas eficientes.

Como conclusión se puede mostrar una evidencia en la existencia de una mayor eficiencia en la generación de rentabilidad de los bancos comerciales en periodo de crisis, en particular para la industria bancaria comercial en México y la industria bancaria comercial en Chile durante el periodo de estudio (2002-2010).

\section{Referencias}

Akhigbe, A., J. Mc Nulty y J. Verbrugge (2001). Small bank loan quality in a deregulated environment: the information advantage hypothesis. Journal of Economics and Business 53: 325-339.

Ariff M. y L. Can (2008). Cost and profit efficiency of Chinese banks: A non-parametric analysis. China Economic Review 19: 260-273.

Athanassopoulos, A. y D. Giokas (2000). The use of data envelopment analysis in banking institutions: evidence from the Commercial Bank of Greece. Interfaces 30 (2): 81-95.

Berger, A. y D. Humphrey (1997). Efficiency of financial institutions: international survey and directions for future research. Working Paper 97-05, Wharton School, University of Pennsylvania.

Charnes, A., W. Cooper, y E. Rhodes (1978). Measuring the efficiency of decision making units. European Journal of Operational Research 2: 429-444.

Clark, J. y T. Siems (2002). X-Efficiency in banking: looking beyond the balance sheet. Journal of Money, Credit and Banking 34: 987-1013.

Drake, L. y B. Howcroft (1994). Relative efficiency in the branch network of a UK bank: an empirical study. Omega 22: 83-90.

Dekker, D. y T. Post (2001). A quasi-concave DEA model with an application for bank branch performance evaluation. Journal of Operational Research 132: 296-311. 
Färe, R., S. Grosskopf y W. Weber (2004). The effect of risk-based capital requirements on profit efficiency in banking. Applied Economics 36: 1731-1743.

Färe, R., S. Grosskopf y C. Lovell (1982). The measurement of efficiency of production. Massachusetts: Kluwer Academic Publishers.

Farrell, M (1957). The measurement of productive efficiency. Journal of the Royal Statistical Society Series A (General) 120: 253-290.

Golany, B. (1988). An interactive MOLP approach for the extension of DEA to effectiveness analysis. Journal of Operational Research Society 39: 725-734.

Hannan, T. (1991). Foundations of the structure-conduct-performance paradigm in banking. Journal of Money, Credit, and Banking 23: 68-84.

Halkos G. y D. Salamoulis (2004). Efficiency measurement of the Greek commercial banks with the use of financial ratios: a data envelopment analysis approach. Management Accounting Research 15: 201-224.

Haslem J., C. Scheraga, y J. Bedingfield (1999). DEA Efficiency profiles of US banks operating internationally. International review of economics and finance 8: 165-182.

Jemric, I. y B. Vujcic (2002). Efficiency of banks in Croatia: A DEA approach. Comparative Economic Studies 44: 169-193.

Maudos, J., J. Pastor, F. Pérez y J. Quesada (2002) Cost and profit efficiency in european banks. Journal of International Financial Markets, Institutions and Money 12: 33-58.

McAllister, P. y D. McManus (1993). Resolving the scale efficiency puzzle in banking. Journal of Banking and Finance 17: 389-405.

Mukherjee, K., S. Ray y S. Miller (2001). Productivity growth in large US commercial banks: the initial post-deregulation experience. Journal of Banking and Finance 25: 913-939. 
Ozkan-Gunay, N. y A. Tektas (2006). Efficiency analysis of the Turkish banking sector in precrisis and crisis period: A DEA approach. Contemporary Economic Policy 24: 418-431.

Purroy, P. y V. Salas (2000). Strategic competition in retail banking under expense preference behavior. Journal of Banking and Finance 24: 809-824.

Rhoades, S. (1980). Monopoly and expense preference behavior: an empirical test of a behavioralist hypothesis. Southern Economic Journal 47: 419-432.

Rogers, K. (1998). Nontraditional activities and the efficiency of US commercial banks. Journal of Banking \& Finance 22: 467-482.

Saha, A. y T. Ravishankar (2000). Rating of Indian Commercial Banks: A DEA Approach. European Journal of Operations Research 124: 187-203.

Sathye, M. (2001). 'X-efficiency in Australian banking: an empirical investigation'. Journal of Banking and Finance 25: 613-630.

Sathye, M. (2003). Efficiency of banks in a developing economy: The case of India. European Journal of Operational Research 148: 662-671.

Seiford L. (1996). Data envelopment analysis: the evaluation of the state of the art (1978-1995). The Journal of Productivity Analysis 9: 99-137.

Sevcovic, D., M. Halicka y P. Brunovsky.(2001). DEA analysis for a large structured bank branch network. Central European Journal of Operations Research 9 (4): 329-342.

Sherman, D. y G. Ladino (1995). Managing Bank Productivity Using Data Envelopment Analysis (DEA). Interfaces 25: 60-73.

Thanassoulis, E. (2003). Introduction to the Theory and Application of Data Envelopment Analysis. A Foundation text with integrated software. 2a. ed. Massachusetts: Kluwer Academic Publishers.

Van Horen, N. (2007). Foreign banks in developing countries; origin matters. Emerging Markets Review 8: 81-105. 
Vassiloglou, M. y D. Giokas (1990). A study of the relative efficiency of bank branches: an application of data envelopment analysis. Journal of Operational Research Society 41: 591-597.

(A) 
ANEXOS

Anexo1

Listado de bancos analizados

\begin{tabular}{|l|l|}
\hline Bancos mexicanos & Bancos chilenos \\
\hline Banamex & Banco Bice \\
\hline Bancen & Banco BilbaoVizcaya Argentaria, Chile \\
\hline Banco Afirme & Banco de Chile \\
\hline Banco Azteca & Banco de Crédito e Inversiones \\
\hline Banco Compartamos & Banco del estado de Chile \\
\hline Banco del Bajio & Banco Santander-Chile \\
\hline Banco Inbursa & Banco Security \\
\hline Banco Interacciones & Bankboston//Banco Itau Chile \\
\hline Banco Santander México & Citibank N.A. \\
\hline Banorte & Corpbanca \\
\hline Banregio & Scotiabank Sudamericano \\
\hline Bansí & Banco del Desarrollo \\
\hline Bbva Bancomer & \\
\hline HSBC & \\
\hline Invex & \\
\hline Ixe & \\
\hline Scotiabank & \\
\hline Serfin & \\
\hline
\end{tabular}

Los bancos comerciales analizados son los principales de cada mercado. Como criterios se utilizaron los que tuvieran utilidades, ya que de lo contrario no son eficiente y no se pueden desarrollar los modelos, también se incluyó un análisis de filtro por nivel de ventas y nivel de activos. En particular sobre el $4 \%$ de participación de mercado y en algún periodo activos sobre diez millones de dólares. 
Anexo 2

DMU eficientes para el caso mexicano periodo de crisis un solo análisis (2008-2010)

\begin{tabular}{|c|c|c|c|c|}
\hline \multirow[t]{2}{*}{ Resultado } & \multicolumn{2}{|c|}{ ROA } & \multicolumn{2}{|r|}{ ROE } \\
\hline & DMU & Eficiencia & DMU & Eficiencia \\
\hline & DMU8 2008 & 0.318 & DMU8 2008 & 0.042 \\
\hline & DMU9 2008 & 0.685 & DMU9 2008 & 0.118 \\
\hline & DMU10 2008 & 0.537 & DMU10 2008 & 0.076 \\
\hline & DMU11 2008 & 0.133 & DMU11 2008 & 0.040 \\
\hline & DMU12 2008 & 0.067 & DMU12 2008 & 0.195 \\
\hline & DMU1 2008 & 0.092 & DMU1 2008 & 0.107 \\
\hline & DMU13 2008 & 0.059 & DMU13 2008 & 0.433 \\
\hline & DMU14 2008 & 0.408 & DMU14 2008 & 0.091 \\
\hline & DMU15 2008 & 0.050 & DMU15 2008 & 0.132 \\
\hline & DMU16 2008 & 1.000 & DMU16 2008 & 1.000 \\
\hline & DMU6 2008 & 0.189 & DMU6 2008 & 0.177 \\
\hline & DMU4 2008 & 0.222 & DMU4 2008 & 0.172 \\
\hline & DMU7 2008 & 0.073 & DMU7 2008 & 0.048 \\
\hline & DMU3 2008 & 0.051 & DMU3 2008 & 0.319 \\
\hline & DMU5 2008 & 0.229 & DMU5 2008 & 0.159 \\
\hline & DMU17 2008 & 0.003 & DMU17 2008 & 0.003 \\
\hline & DMU8 2009 & 0.454 & DMU8 2009 & 0.062 \\
\hline & DMU9 2009 & 0.539 & DMU9 2009 & 0.103 \\
\hline & DMU10 2009 & 0.678 & DMU10 2009 & 0.108 \\
\hline & DMU12 2009 & 0.431 & DMU12 2009 & 0.116 \\
\hline & DMU1 2009 & 0.184 & DMU1 2009 & 0.193 \\
\hline & DMU13 2009 & 0.095 & DMU13 2009 & 0.542 \\
\hline & DMU14 2009 & 0.298 & DMU14 2009 & 0.066 \\
\hline & DMU15 2009 & 0.034 & DMU15 2009 & 0.096 \\
\hline & DMU16 2009 & 1.000 & DMU16 2009 & 0.940 \\
\hline & DMU4 2009 & 0.227 & DMU4 2009 & 0.143 \\
\hline & DMU7 2009 & 0.046 & DMU7 2009 & 0.223 \\
\hline & DMU3 2009 & 0.062 & DMU3 2009 & 0.341 \\
\hline & DMU5 2009 & 0.203 & DMU5 2009 & 0.147 \\
\hline & DMU17 2009 & 0.009 & DMU17 2009 & 0.044 \\
\hline & DMU8 2010 & 0.503 & DMU8 2010 & 0.066 \\
\hline & DMU9 2010 & 0.673 & DMU9 2010 & 0.112 \\
\hline & DMU10 2010 & 0.594 & DMU10 2010 & 0.136 \\
\hline & DMU11 2010 & 0.221 & DMU11 2010 & 0.006 \\
\hline & DMU12 2010 & 0.045 & DMU12 2010 & 0.124 \\
\hline & DMU1 2010 & 0.160 & DMU1 2010 & 0.169 \\
\hline & DMU13 2010 & 0.056 & DMU13 2010 & 0.353 \\
\hline & DMU14 2010 & 0.378 & DMU14 2010 & 0.089 \\
\hline & DMU15 2010 & 0.039 & DMU15 2010 & 0.103 \\
\hline & DMU16 2010 & 1.000 & DMU16 2010 & 0.814 \\
\hline & DMU4 2010 & 0.326 & DMU4 2010 & 0.212 \\
\hline & DMU7 2010 & 0.034 & DMU7 2010 & 0.169 \\
\hline & DMU3 2010 & 0.050 & DMU3 2010 & 0.287 \\
\hline & DMU5 2010 & 0.255 & DMU5 2010 & 0.165 \\
\hline & DMU17 2010 & 0.016 & DMU17 2010 & 0.065 \\
\hline & DMU6 (2004) & 0.725 & DMU2 (2002) & 1.000 \\
\hline & DMU2(2005) & 1.000 & DMU4 (2003) & 0.773 \\
\hline & & & DMU5 (2003) & 0.911 \\
\hline & & & DMU2(2005) & 1.000 \\
\hline
\end{tabular}


Anexo 3

DMU eficientes para el caso chileno periodo de crisis un solo análisis (2008-2010)

\begin{tabular}{ccc|cc}
\hline Resultado & \multicolumn{2}{c|}{ ROA } & & ROE \\
\hline DMU & Eficiencia & DMU & Eficiencia \\
\hline DMU1 2008 & 0.227 & DMU1 2008 & 0.296 \\
DMU2 2008 & 0.402 & DMU2 2008 & 0.394 \\
DMU3 2008 & 0.316 & DMU3 2008 & 0.267 \\
DMU4 2008 & 0.298 & DMU4 2008 & 0.315 \\
DMU5 2008 & 0.346 & DMU5 2008 & 0.403 \\
DMU6 2008 & 0.455 & DMU6 2008 & 0.449 \\
DMU7 2008 & 0.197 & DMU7 2008 & 0.221 \\
DMU9 2008 & 0.178 & DMU9 2008 & 0.294 \\
DMU10 2008 & 0.257 & DMU10 2008 & 0.227 \\
DMU1 2009 & 0.313 & DMU1 2009 & 0.368 \\
DMU2 2009 & 0.495 & DMU2 2009 & 0.705 \\
DMU3 2009 & 0.455 & DMU3 2009 & 0.433 \\
DMU4 2009 & 0.328 & DMU4 2009 & 0.294 \\
DMU5 2009 & 0.329 & DMU5 2009 & 0.357 \\
DMU6 2009 & 0.590 & DMU6 2009 & 0.551 \\
DMU7 2009 & 0.175 & DMU7 2009 & 0.334 \\
DMU8 2009 & 0.082 & DMU8 2009 & 0.083 \\
DMU9 2009 & 0.232 & DMU9 2009 & 0.318 \\
DMU10 2009 & 0.334 & DMU10 2009 & 0.368 \\
DMU1 2010 & 0.371 & DMU1 2010 & 0.376 \\
DMU2 2010 & 0.989 & DMU2 2010 & $\mathbf{1 . 0 0 0}$ \\
DMU3 2010 & 0.950 & DMU3 2010 & $\mathbf{1 . 0 0 0}$ \\
DMU4 2010 & 0.411 & DMU4 2010 & 0.381 \\
DMU5 2010 & 0.535 & DMU5 2010 & 0.469 \\
DMU6 2010 & $\mathbf{1 . 0 0 0}$ & DMU6 2010 & 0.888 \\
DMU7 2010 & 0.636 & DMU7 2010 & 0.757 \\
DMU8 2010 & 0.172 & DMU8 2010 & 0.168 \\
DMU9 2010 & 0.255 & DMU9 2010 & 0.309 \\
DMU10 2010 & 0.566 & DMU10 2010 & 0.470 \\
DMU3 (2003) & 0.644 & DMU1 (2002) & 0.586 \\
DMU7 (2003) & 0.737 & DMU7 (2003) & 0.974 \\
DMU3 (2005) & $\mathbf{1 . 0 0 0}$ & DMU3 (2005) & 0.560 \\
\hline & & &
\end{tabular}


Anexo 4.

Gráficos de principales variables económicas de México

Figura 2

Evolución de la variación anual del PIB mexicano

\% Variación

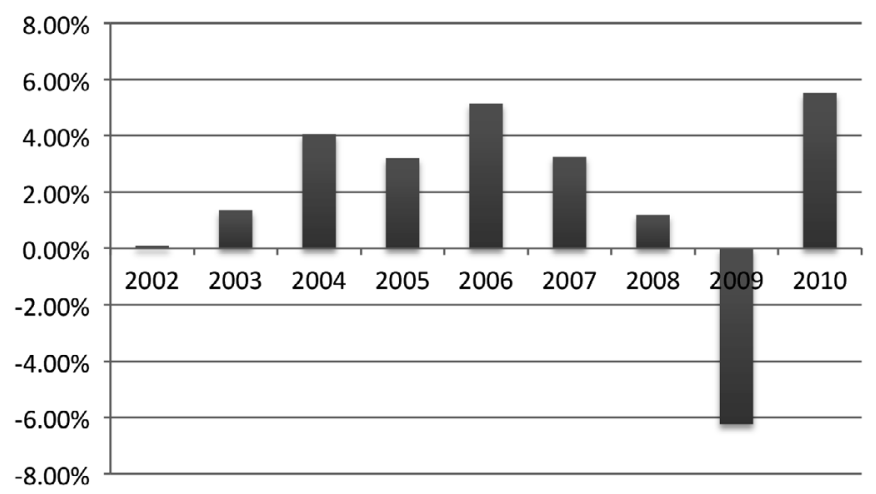

Fuente: Elaboración propia con base en estadísticas del Instituto Nacional de Estadísticas y Geografía de México, INEGI.

Figura 3

Evolución de la inflación anual mexicana

\% Variación

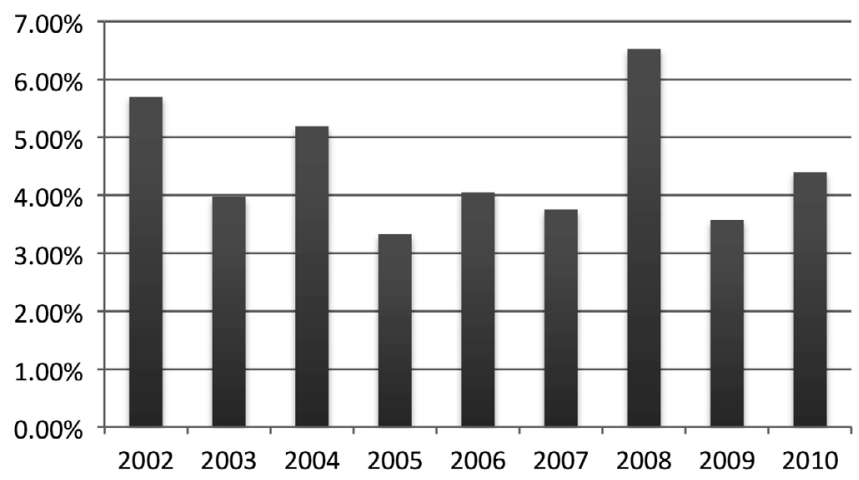

Fuente: Elaboración propia con base en las estadísticas del Instituto Nacional de Estadísticas y Geografía de México, INEGI. 


\section{Figura 4}

Evolución mensual del tipo de cambio, pesos mexicanos por dólar estadounidense

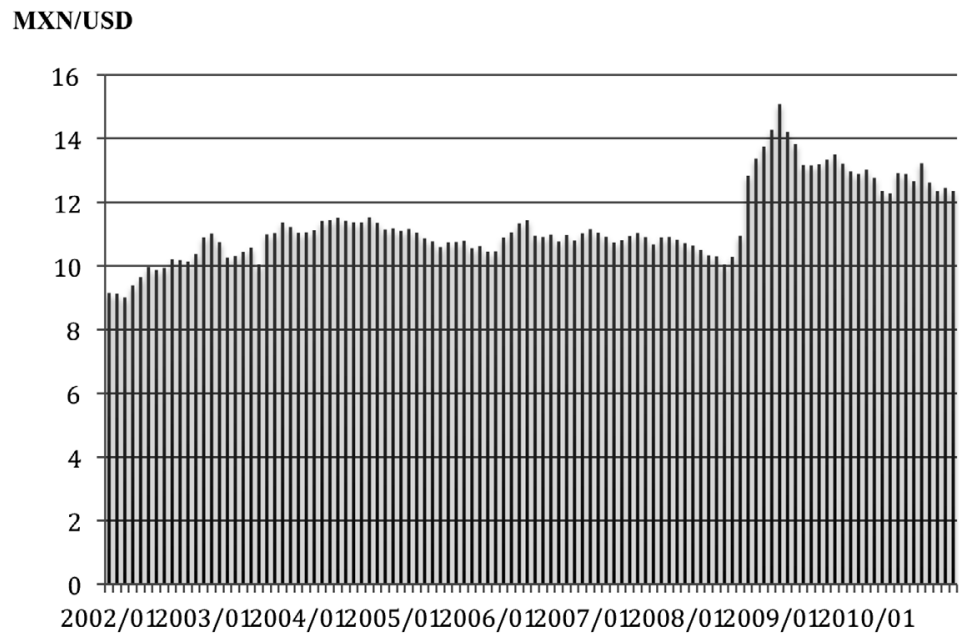

Fuente: Elaboración propia con base en estadísticas del Instituto Nacional de Estadísticas y Geografía de México, INEGI.

\section{Anexo 5. Gráficos de principales variables económicas de Chile}

\section{Figura 5}

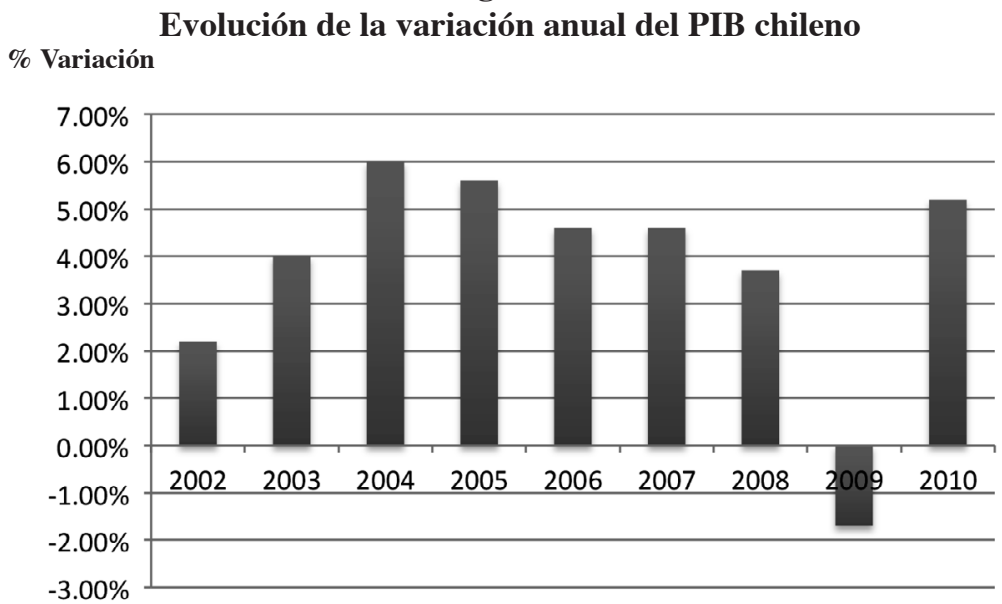

Fuente: Elaboración propia con base en estadísticas del Banco Central de Chile. 


\section{Figura 6}

\section{Evolución de la inflación anual chilena}

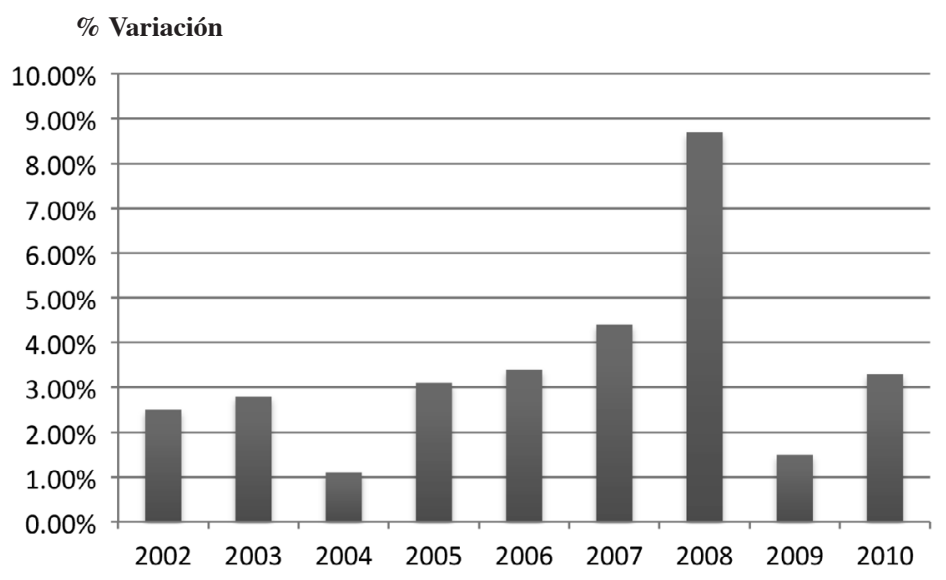

Fuente: Elaboración propia con base en las estadísticas del Banco Central de Chile.

Figura 7

Evolución mensual del tipo de cambio, pesos chilenos por dólar estadounidense

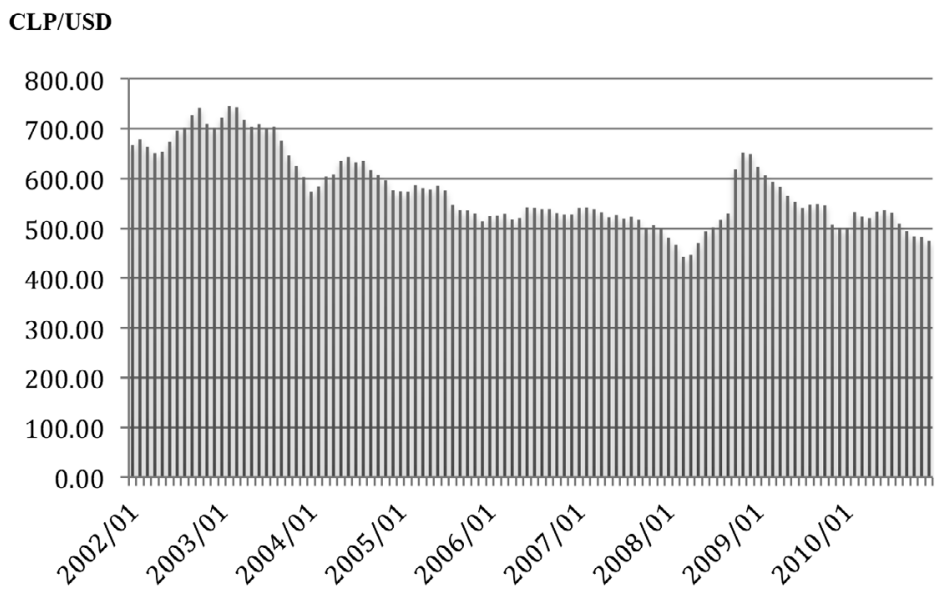

Fuente: Elaboración propia con base en las estadísticas del Banco Central de Chile. 